\title{
The TRIUMF nuclear structure program and TIGRESS
}

P. E. Garrett, A. Andreyev, R. A. E.Austin, G. C. Ball, D. Bandyopadhyay, J. A. Becker, A.J. Boston, H.C. Boston, R. S. Charkrawarthy, R, Churchman, D. Cline, R. J. Cooper, D. Cross, D. Dashdorj, G. A. Demand, M. R. Dimmock, T. Drake, P. Finlay, K. Gagnon, A. T. Gallant, K. L. Green, A. N. Grint, G. F. Grinyer, G. Hackman, L. J. Harkness, A. B. Hayes, R. Kanungo, W. D. Kulp, K. G. Leach, G. Lee, J. R. Leslie, R. Maharaj, J. P. Martin, C. Mattoon, W. J. Mills, A. C. Morton, L. Nelson, O. Newman, P. J. Nolan, E. Padilla-Rodal, C. J. Pearson, A. A. Phillips, M. Porter-Peden, J. J. Ressler, C. Ruiz, F. Sarazin, M. A. Schumaker, D. P. Scraggs, M. T. Strange, M. Subramanian, C. E. Svensson, J. C. Waddington, J. Wan, A. Whitebeck, S. J. Williams, J. L. Wood, J. C. Wong, C. Y. Wu, E. F. Zganjar

\section{September 5, 2006}

19th International Conference on the Application of Accelerators in Research and Industry Fort Worth, TX, United States August 20, 2006 through August 25, 2006 
This document was prepared as an account of work sponsored by an agency of the United States Government. Neither the United States Government nor the University of California nor any of their employees, makes any warranty, express or implied, or assumes any legal liability or responsibility for the accuracy, completeness, or usefulness of any information, apparatus, product, or process disclosed, or represents that its use would not infringe privately owned rights. Reference herein to any specific commercial product, process, or service by trade name, trademark, manufacturer, or otherwise, does not necessarily constitute or imply its endorsement, recommendation, or favoring by the United States Government or the University of California. The views and opinions of authors expressed herein do not necessarily state or reflect those of the United States Government or the University of California, and shall not be used for advertising or product endorsement purposes. 
ID 478

\title{
The TRIUMF Nuclear Structure Program and TIGRESS
}

\author{
P.E. Garrett ${ }^{1,2 *}$, A. Andreyev ${ }^{3}$, R.A.E. Austin ${ }^{4}$, G.C. Ball ${ }^{2}$, D. Bandyopadhyay ${ }^{1}$, J.A. Becker ${ }^{5}$,
} A.J. Boston ${ }^{6}$, H.C. Boston ${ }^{6}$, R.S. Chakrawarthy ${ }^{3}$, R. Churchman ${ }^{2}$, D. Cline ${ }^{7}$, R.J. Cooper ${ }^{6}$, D. Cross $^{3}$, D. Dashdorj ${ }^{8}$, G.A. Demand ${ }^{1}$, M.R. Dimmock ${ }^{6}$, T. Drake ${ }^{9}$, P. Finlay ${ }^{1}$, K. Gagnon ${ }^{3}$, A.T. Gallant ${ }^{4}$, K.L. Green ${ }^{1}$, A.N. Grint ${ }^{6}$, G.F. Grinyer ${ }^{1}$, G. Hackman ${ }^{2}$, L.J. Harkness ${ }^{2,6}$, A.B. Hayes ${ }^{7}$, R. Kanungo ${ }^{2}$, W.D. Kulp ${ }^{10}$, K.G. Leach ${ }^{1}$, G. Lee ${ }^{2}$, J.R. Leslie ${ }^{11}$, R. Maharaj ${ }^{2}$, J-P. Martin ${ }^{12}$, C. Mattoon ${ }^{13}$, W.J. Mills ${ }^{3}$, A.C. Morton ${ }^{2}$, L. Nelson ${ }^{6}$, O. Newman ${ }^{2}$, P.J. Nolan ${ }^{6}$, E. Padilla-Rodal ${ }^{2}$, C.J. Pearson ${ }^{2}$, A.A. Phillips ${ }^{1}$, M. Porter-Peden ${ }^{13}$, J.J. Ressler ${ }^{3}$, C. Ruiz ${ }^{2}$,

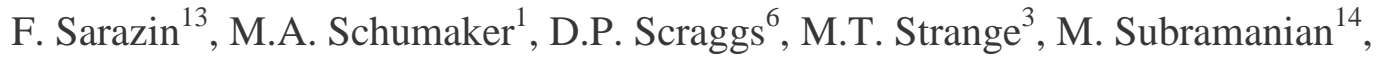
C.E. Svensson ${ }^{1}$, J.C. Waddington ${ }^{15}$, J. Wan ${ }^{3}$, A. Whitbeck ${ }^{7}$, S.J. Williams ${ }^{2}$, J.L. Wood ${ }^{10}$, J.C. Wong ${ }^{1}$, C.Y. Wu ${ }^{5}$, E.F. Zganjar ${ }^{16}$ 1) Department of Physics, University of Guelph, Guelph, ON N1G2W1, Canada 2) TRIUMF, 4004 Wesbrook Mall, Vancouver, BC, V6T2A3 Canada 3) Simon Frasier University, Burnaby, BC, V5A1S6 Canada 4) Dept. of Astronomy and Physics, Saint Mary's University, Halifax, NS B3H 3C3 Canada 5) Lawrence Livermore National Laboratory, 7000 East Ave., Livermore, CA, 94551 USA 6) Department of Physics, University of Liverpool, Liverpool, L69 7ZE UK 7) Department of Physics and Astronomy, University of Rochester, Rochester, NY, 14627 USA 8) Department of Physics, North Carolina State University, Raleigh, NC 27695 USA 9) Department of Physics, University of Toronto, Toronto, ON M5S1A7 Canada 10) Department of Physics, Georgia Institute of Technology, Atlanta, GE, USA

\footnotetext{
${ }^{*}$ Corresponding author: P.E. Garrett, Department of Physics, University of Guelph, Guelph, ON N1G2W1 Canada E-mail: pgarrett@physics.uoguelph.ca

Tel: 001-519-824-4120 ext. 52192

FAX: 001-519-836-9967
} 
ID 478

11) Department of Physics, Queens University, Kingston, ON K7L 3N6 Canada

12) Département de Physique, Université de Montréal, Montréal, QC H3C3J7 Canada 13) Department of Physics, Colorado School of Mines, Golden, CO 80401 USA 14) Dept. of Physics and Astronomy, University of British Columbia, Vancouver, BC V6T1Z1 Canada

\section{5) Dept. of Physics and Astronomy, McMaster University, Hamilton, ON L8S4M1 Canada}

16) Dept. of Physics and Astronomy, Louisiana State University, Baton Rouge, LA, 70803 USA

\section{ABSTRACT}

The Isotope Separator and Accelerator (ISAC) facility located at the TRIUMF laboratory in Vancouver, Canada, is one of the world's most advanced ISOL-type radioactive ion beam facilities. An extensive $\gamma$-ray spectroscopy programme at ISAC is centred around two major research facilities: i) the $8 \pi \gamma$-ray spectrometer for $\beta$-delayed $\gamma$-ray spectroscopy experiments with the low-energy beams from ISAC-I, and ii) the next-generation TRIUMF-ISAC GammaRay Escape Suppressed Spectrometer (TIGRESS) for in-beam experiments with the accelerated radioactive ion beams. An overview of these facilities and recent results from the diverse programme of nuclear structure and fundamental interaction studies they support is presented.

\section{INTRODUCTION}

Nuclear physics has undergone a renaissance due to the development of new radioactive-ion beam facilities at several laboratories around the world. Of these, the Isotope Separator and ACcelerator facility (ISAC), which is of the ISOL type, located at TRIUMF in Vancouver, Canada, currently has the highest beam power available worldwide at $50 \mathrm{~kW}$. ISAC employs a $500 \mathrm{MeV}$ proton beam, delivered by a cyclotron, with currents up to $100 \mu \mathrm{A}$ on targets ranging from SiC to Ta. Some of the most notable achievements are beams of ${ }^{11} \mathrm{Li}$ up to $5 \times 10^{4} \mathrm{~s}^{-1},{ }^{26} \mathrm{Al}$ 


\section{ID 478}

1 at $3 \times 10^{10} \mathrm{~s}^{-1}$, the separation of isomeric from ground state Ag beams with the laser ion source,

2 and the observation of $t_{1 / 2}=3.1 \mathrm{~ms}{ }^{179 m} \mathrm{Lu}$ beam released from the Ta target. The ISAC facility

3 (hereafter referred to as ISAC-I) is able to provide mass-separated beams at low-energy

4 (typically $30 \mathrm{keV}$ ) as well as accelerated up to approximately $1.7 \mathrm{MeV} /$ nucleon and mass 30. An

5 extension of the laboratory, known as ISAC-II, will be able to accelerate beams up to mass 150

6 and approximately $6.5 \mathrm{MeV} /$ nucleon when completed. The wide variety of intense radioactive

7 beams provides for a full programme of nuclear astrophysics, nuclear structure, weak interaction

8 tests, and materials science. In the present work, the focus is on the nuclear structure programme

9 using the $8 \pi$ spectrometer at ISAC-I and the TIGRESS spectrometer that will be located at

10 ISAC-II.

\section{NUCLEAR STRUCTURE AT ISAC-I}

12 The nuclear structure programme at ISAC-I is centred (although not exclusively) on the $8 \pi$

13 spectrometer and its auxiliary detectors. The $8 \pi$ spectrometer consists of twenty HPGe detectors

14 with a relative efficiency of 20-25\% surrounded by annular BGO shields for Compton

15 suppression and a BGO "back-plug" detector. The detectors are positioned at the hexagon

16 positions of a truncated icosahedron. The radioactive beam typically at $30-\mathrm{keV}$ energy is

17 deposited in the centre of the array, on either a metallic target or on a moving tape collector

18 (MTC). The target-to-detector distance is approximately $14 \mathrm{~cm}$, and the array efficiency is

$19 \sim 1.5 \%$ at $1.33 \mathrm{MeV}$. The tape of the MTC forms a continuous loop, typically $120 \mathrm{~m}$ in length,

20 and has its movement governed by the programming of a controller unit.

21 A number of auxiliary detectors systems have been implemented in the $8 \pi$ spectrometer;

22 SCEPTAR - the SCintillating Electron Positron Tagging ARray, PACES - the Pentagonal Array

23 for Conversion Electron Spectroscopy, and DANTE - the Dipentagonal Array for Nuclear 
1 Timing Experiments. SCEPTAR consists of twenty pieces of $1.6 \mathrm{~mm}$ thick plastic scintillator

2 surrounding the target position, arranged so that there is a one-to-one match with the Ge

3 detectors. Being relatively thin, SCEPTAR acts as a $\Delta E$ detector for electrons and positrons, and

4 is useful to indicate that a $\beta$ decay has occurred. PACES incorporates five $\mathrm{Si}(\mathrm{Li})$ detectors $5 \mathrm{~mm}$

5 thick that replace the upstream half of SCEPTAR, and is most useful for conversion electron

6 spectroscopy. DANTE consists of ten $\mathrm{BaF}_{2}$ detectors positioned in the open pentagonal

7 positions of the truncated icosahedron and is used for fast-timing measurements. This

8 combination of detector systems is unique in the world, and provides for comprehensive

9 spectroscopy of nuclei following $\beta$ decay.

10 The $8 \pi$ nuclear structure program has examined nuclei from ${ }^{11} \mathrm{Li}$ to ${ }^{179} \mathrm{Lu}$. An initial study

11 [1] with the $8 \pi$ array to observe the $\beta$ decay of a beam of $10^{3}{ }^{11} \mathrm{Li} \mathrm{s}^{-1}$ suggested that the

12 theoretical prediction of a $\beta$ decay occurring in the core and leaving intact the halo neutrons

13 could be realized. Further studies [2] with greatly increased sensitivity using $8 \pi+$ SCEPTAR and

14 a beam of $>10^{4}{ }^{11} \mathrm{Li} \mathrm{s}^{-1}$ have been performed that will yield a much more detailed picture. Figure

151 shows portions of spectra obtained from the two ${ }^{11} \mathrm{Li}$ experiments. The improvement in quality

16 and statistics of the data is obvious, and the detailed $\gamma$-ray lines-shapes now available allow for

17 precise determination of the level life-times and neutron decay braches. Future studies in this

18 direction are planned, such as the heavier halo system ${ }^{14} \mathrm{Be}$.

19 A number of theoretical calculations predict that the familiar shell gaps that give rise to the

20 "magic" numbers, or closed major shells, may change drastically in neutron-rich nuclei, and thus

21 a major line of research is the mapping of the nuclear shells as one proceeds away from stability.

22 The investigation of shell structure in the neutron-rich $\mathrm{Mg}$ and $\mathrm{O}$ isotopes is particularly

23 interesting due to the "island of inversion" where the changes in the shell structure cause the 
ID 478

1 disappearance of the $N=20$ neutron shell with the consequence that nuclei become deformed

2 rather than retain a spherical shape, and other nuclei that would normally be predicted to be

3 bound are unbound. An initial experiment [3] examining the decay of ${ }^{32} \mathrm{Na}$ demonstrated that

4 spectroscopy is possible with beam intensities as low as $1 \mathrm{~s}^{-1}$. More detailed studies must await

5 the development of the actinide target for an expected 2-3 orders-of-magnitude boost in beam

6 intensity. The very-neutron rich $\mathrm{Cd}$ and $\mathrm{Pd}$ isotopes are of interest in order to probe the

7 quenching of the $N=82$ shell below ${ }^{132} \mathrm{Sn}$. This has an important consequence on observed

8 elemental abundances, particularly on the abundance of nuclei in the mass 120 region [4-6].

9 This programme will soon commence now that $\mathrm{Ag}$ beams have been developed. A further

10 region of interest, due to predicted shell closures at $N=32$ and $N=34$, is the neutron-rich $\mathrm{Ca}$

11 isotopes and an experiment was recently performed to observe the $\beta$ decay of the ${ }^{52} \mathrm{~K}$. The ${ }^{52} \mathrm{~K}$

12 beam intensity was $\sim 10 \mathrm{~s}^{-1}$, and sufficient data were collected to permit $\gamma \gamma$ coincidences, analysis

13 of which is ongoing [7].

14 For many years it has been known that nuclei near $N=90$ exhibit a rapid change in the shape

15 of the ground state as the neutron number increases from $N=88$ to $N=92$. The excited-state

16 spectrum also undergoes a rapid evolution from that resembling a vibrational system to a

17 rotational one. Very recently [8,9], based on improved spectroscopy through ${ }^{152}$ Eu decay and

18 IBM calculations, it was suggested that nuclei at $N=90$ are at a critical point of a shape phase

19 transition. A new model, based on a solution of the Bohr Hamiltonian with a square-well

20 potential in the $\beta$ shape degree of freedom, has been developed centred on this idea [10]. It has

21 been claimed [11] that the new model successfully describes the level structures of a series of

$22 N=90$ isotones; however, the lack of precision data prevents a definitive conclusion. 
1 Studies to date using the $8 \pi$ spectrometer to investigate $N=90$ nuclei include ${ }^{156} \mathrm{Ho},{ }^{158} \mathrm{Tm}$,

2 and ${ }^{160} \mathrm{Lu}$ decay. Beam intensities for these experiments can reach $>10^{7} \mathrm{~s}^{-1}$, and typically data on

3 the order of $1 \mathrm{~TB}$ are collected. New EO transitions have been observed that are critical in the

4 determination of shape coexistence in these nuclei, but analysis of the data is not complete thus

$5 \rho^{2}(E O)$ values are not yet available. Figure 2 displays portions of the $\gamma$-ray and conversion

6 electron spectrum collected with one $8 \pi$ Ge detector and one of the PACES Si detectors.

7 Comparison of the intensities of the electron lines to those in the $\gamma$-ray spectrum allows the

8 determination of the multipolarity of the transition.

\section{NUCLEAR STRUCTURE WITH TIGRESS}

10 Many of the experiments performed at ISAC-II, with its ability of accelerating the

11 radioactive beams beyond the Coulomb barrier for a wide variety of targets, will involve inverse

12 kinematics reactions. For $\gamma$-ray detection, it is important to have very-high photo-peak efficiency

13 while incorporating the ability to locate the first interaction point precisely so that a proper

14 Doppler shift correction can be applied. It is for this purpose that the TRIUMF-ISAC Gamma-

15 Ray Escape Suppressed Spectrometer (TIGRESS) is being constructed.

16 TIGRESS, when fully completed in 2009, will incorporate twelve highly segmented clover

17 detectors mounted in a frame with the geometry of a rhombicuboctahedron. Each crystal in a

18 clover detector has approximately $40 \%$ relative efficiency, and the outer contacts are segmented

19 4-fold axially and 2-fold longitudinally [12]. A segmented BGO suppression shield consisting of

204 side plates and 4 front shields, and a CsI "back-plug" surround each clover detector for

21 Compton suppression. The eight outer contacts and one core contact are read out with 100-MHz

22 waveform digitizers custom built for TIGRESS, and a waveform analysis demonstrates that

23 position sensitivity of better than $2 \mathrm{~mm}$ can be achieved [13]. There are two standard 
ID 478

1 configurations for the clover detectors in TIGRESS; a "fully-forward" high efficiency mode, in

2 which the front BGO shields are pulled back to allow the Ge detectors to be moved forward, and

3 "fully-suppressed" optimum peak-to-total mode. Detailed GEANT4 simulations [14] indicate

4 that the full array will achieve a photo-peak efficiency of $17 \%$ for a single $\gamma$-ray at $1 \mathrm{MeV}$.

5 A number of auxiliary detectors are planned for TIGRESS. The first to be commissioned is

6 BAMBINO, which consists of 150- $\mu$ m thick Si CD-S2 detectors from Micron Technology Inc.

7 that will be used for light-ion Coulomb excitation. Two such detectors can be mounted $3 \mathrm{~cm}$

8 from the target for both forward and backward hemispheres, covering a solid angle of $\sim 1.15 \pi \mathrm{sr}$,

9 and each have 24 rings in $\theta$ for angles between $20^{\circ}$ and $49^{\circ}$ and between $131^{\circ}$ and $160^{\circ}$, and 16

10 sectors in $\phi$ for $360^{\circ}$ coverage. Figure 3 shows a photograph of BAMBINO in its target chamber

11 during a commissioning experiment. Also planned is a $\mathrm{Si} \Delta E-E$ detector in a box-like geometry

12 to be used for Coulomb excitation studies, single-nucleon transfer, and inelastic scattering, a

13 segmented Bragg detector to be able to identify ions on an event-by-event basis, a CsI detector

14 for light-ion identification for Coulomb excitation and fusion-evaporation studies, and a neutron

15 detector employing deuterated liquid scintillator. A very powerful complement is the

16 ElectroMagnetic Mass Analyzer (EMMA), a recoil spectrometer of the electric-dipole -

17 magnetic-dipole - electric-dipole type [15], that is expected to be available in 2009.

18 The first experiment, performed at ISAC-I, conducted using TIGRESS and BAMBINO was

19 the Coulomb excitation of ${ }^{20,21} \mathrm{Na}$. The system was commissioned with a beam of stable ${ }^{21} \mathrm{Ne}$.

20 The beams had energies of $1.7 \mathrm{MeV} /$ nucleon and intensities of $2-6 \times 10^{6} \mathrm{~s}^{-1}$, and impinged on a

21 target of ${ }^{\text {nat }} \mathrm{Ti}$ that was $450 \mu \mathrm{g} / \mathrm{cm}^{2}$ thick. The resulting $\gamma$-ray spectra for ${ }^{21} \mathrm{Ne}$ and ${ }^{21} \mathrm{Na}$ beams

22 (with a preliminary Doppler correction and without background subtraction) obtained in

23 coincidence with particles detected in BAMBINO are shown in Fig. 4. Of particular interest is 
1 the appearance of the $511-\mathrm{keV}$ annihilation line that arises from the $\beta^{+}$decay of ${ }^{21} \mathrm{Na}$ beam

2 particles; the weakness of the peak indicates that the amount of scattered beam in the target

3 chamber is well under control and will not interfere with the extraction of the matrix elements.

4 By analyzing the particle- $\gamma$ coincidence yield as a function of scattering angle, the matrix

5 element for Coulomb excitation can be uniquely determined.

\section{CONCLUSIONS}

7 Highlights of the nuclear structure studies at the TRIUMF ISAC facility were outlined. The

$88 \pi$ spectrometer and its associated auxiliary detectors is a world unique facility for nuclear

9 physics utilizing $\beta$ decay, in that $\gamma$-ray singles, coincidences, conversion electrons, $\beta$-particle

10 tagging, and lifetime measurements can be performed simultaneously. A broad programme of

11 nuclear structure, nuclear astrophysics, and Standard Model tests is underway. Nuclear structure

12 investigations with TIGRESS have just commenced with the Coulomb excitation of radioactive

$13 \quad{ }^{21} \mathrm{Na}$, and preliminary spectra from the experiment have been presented.

\section{ACKNOWLEDGEMENTS}

15 This work has been supported by the Natural Sciences and Engineering Research Council

16 and the National Research Council (Canada), the U.S. Department of Energy and National

17 Science Foundation, the Sciences and Engineering Research Council (UK). This work was

18 performed in part under the auspices of the U.S. Department of Energy by University of

19 California, Lawrence Livermore National Laboratory under contract No. W-7405-ENG-48.

\section{REFERENCES}

21 [1] F. Sarazin et al., Phys. Rev. C 69 (2004) 031302(R).

22 [2] F. Sarazin et al., to be published.

23 [3] C. Mattoon et al., to be published. 


\section{ID 478}

1 [4] K.-L. Kratz, J.P. Bitouzet, F.-K. Thielmann, P. Möller, B. Pfeiffer, Ap. J. 403 (1993) 216.

2 [5] J. Dobaczewski, I. Hammamoto, W. Nazarewicz, J.A. Sheikh, Phys. Rev. Lett. 72 (1994)

3981.

4 [6] T. Kautzsch et al., Eur. Phys. J. A9 (2000) 201.

5 [7] R.V.F. Janssens and P. Mantica, private communication (2006).

6 [8] R.F. Casten et al., Phys. Rev. C 57 (1998) R1553.

7 [9] I. Iachello, N.V. Zamfir, R.F. Casten, Phys. Rev. Lett. 81 (1998) 1191.

8 [10] I. Iachello, Phys. Rev. Lett. 87 (2001) 052502.

9 [11] R.F. Casten, N.V. Zamfir, Phys. Rev. Lett. 87 (2001) 052503.

10 [12] H.C. Scraggs et al., Nucl. Instrum. Meth. A543 (2005) 431.

11 [13] C.E. Svensson et al., Nucl. Instrum. Meth. A540 (2005) 348.

12 [14] M.A. Schumaker et al., Nucl. Instrum. Meth. A (2005) submitted.

13 [15] B. Davids, C.N. Davids, Nucl. Instrum. Meth. A544 (2005) 565. 


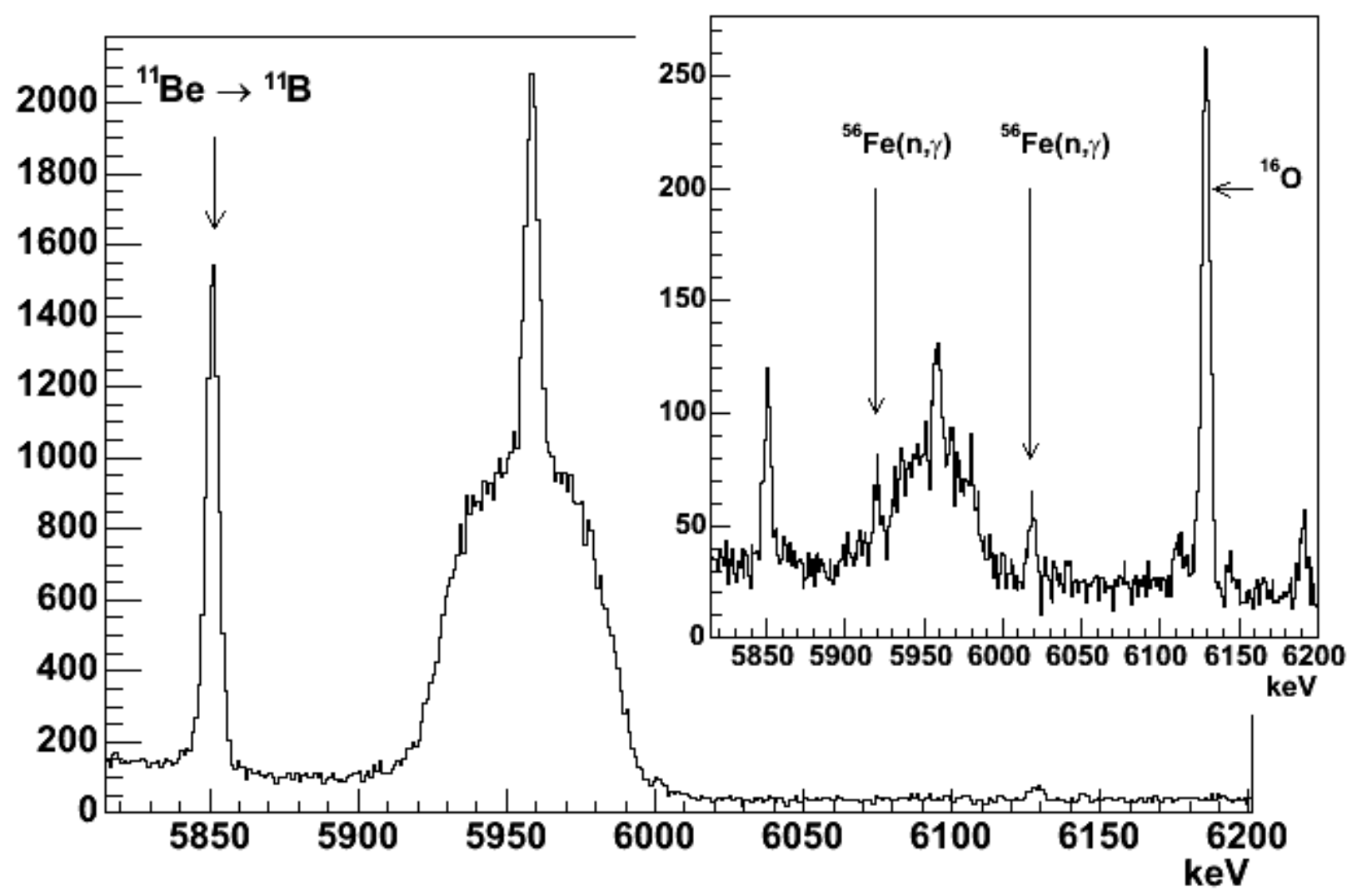

1 Figure 1. Comparison of data obtained from two ${ }^{11} \mathrm{Li}$ decay experiments. In the first experiment

2 (inset), the ${ }^{11} \mathrm{Li}$ beam intensity was $\sim 10^{3} \mathrm{~s}^{-1}$, and only the $8 \pi$ Ge detectors were used. In the

3 second experiment, the ${ }^{11} \mathrm{Li}$ beam intensity was $\sim 1.5 \times 10^{4} \mathrm{~s}^{-1}$, and coincidences with $\beta$ particles

4 detected with SCEPTAR was employed. 

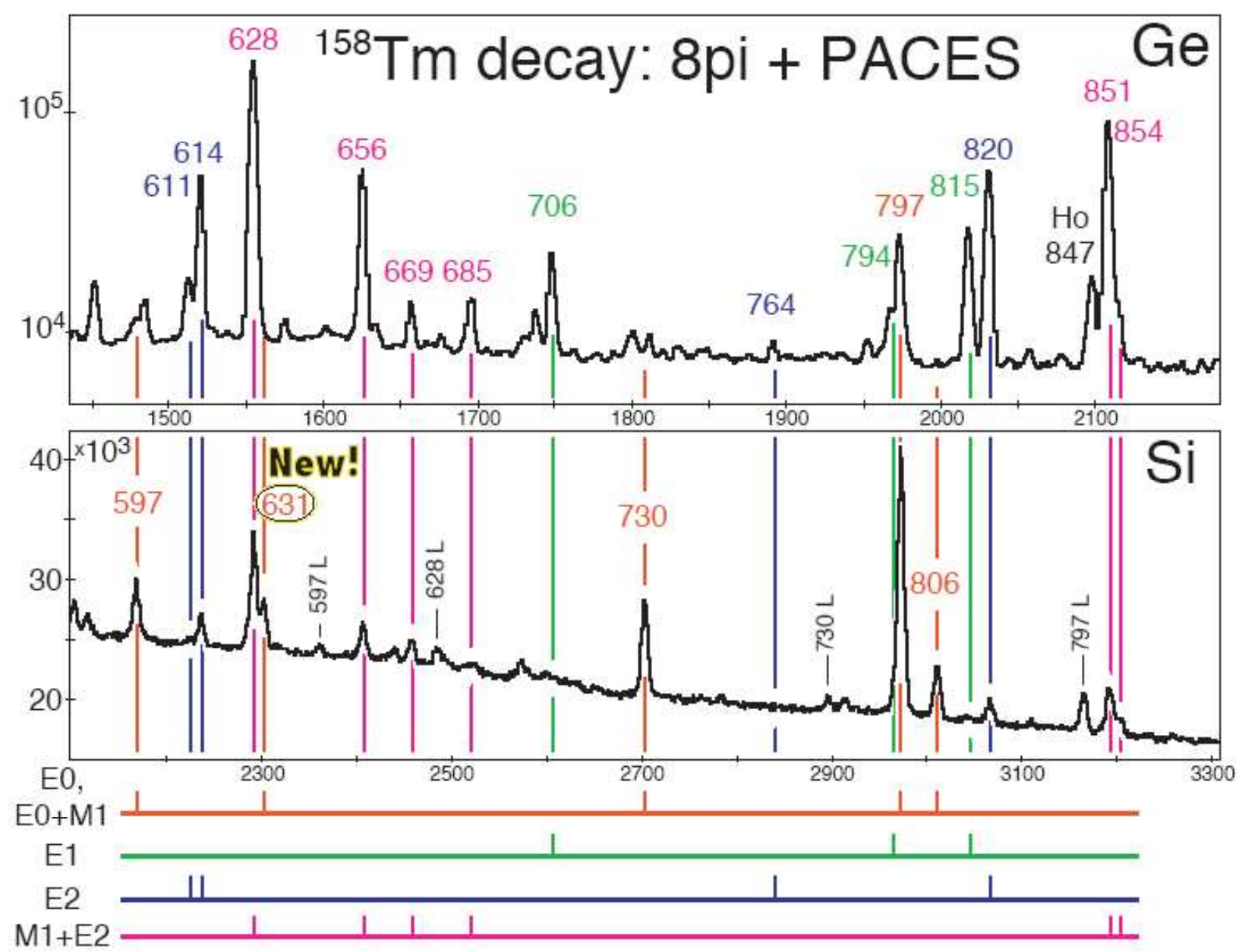

1 Figure 2. Portions of the $\gamma$-ray (top) and electron (bottom) spectra obtained with one of the $8 \pi \mathrm{Ge}$

2 detectors and one of the PACES Si detectors in the decay of ${ }^{158} \mathrm{Tm}$. Peaks that are intense on the

3 electron spectrum but moderate or missing in the $\gamma$-ray spectrum are identified as $E 0$ transitions. 


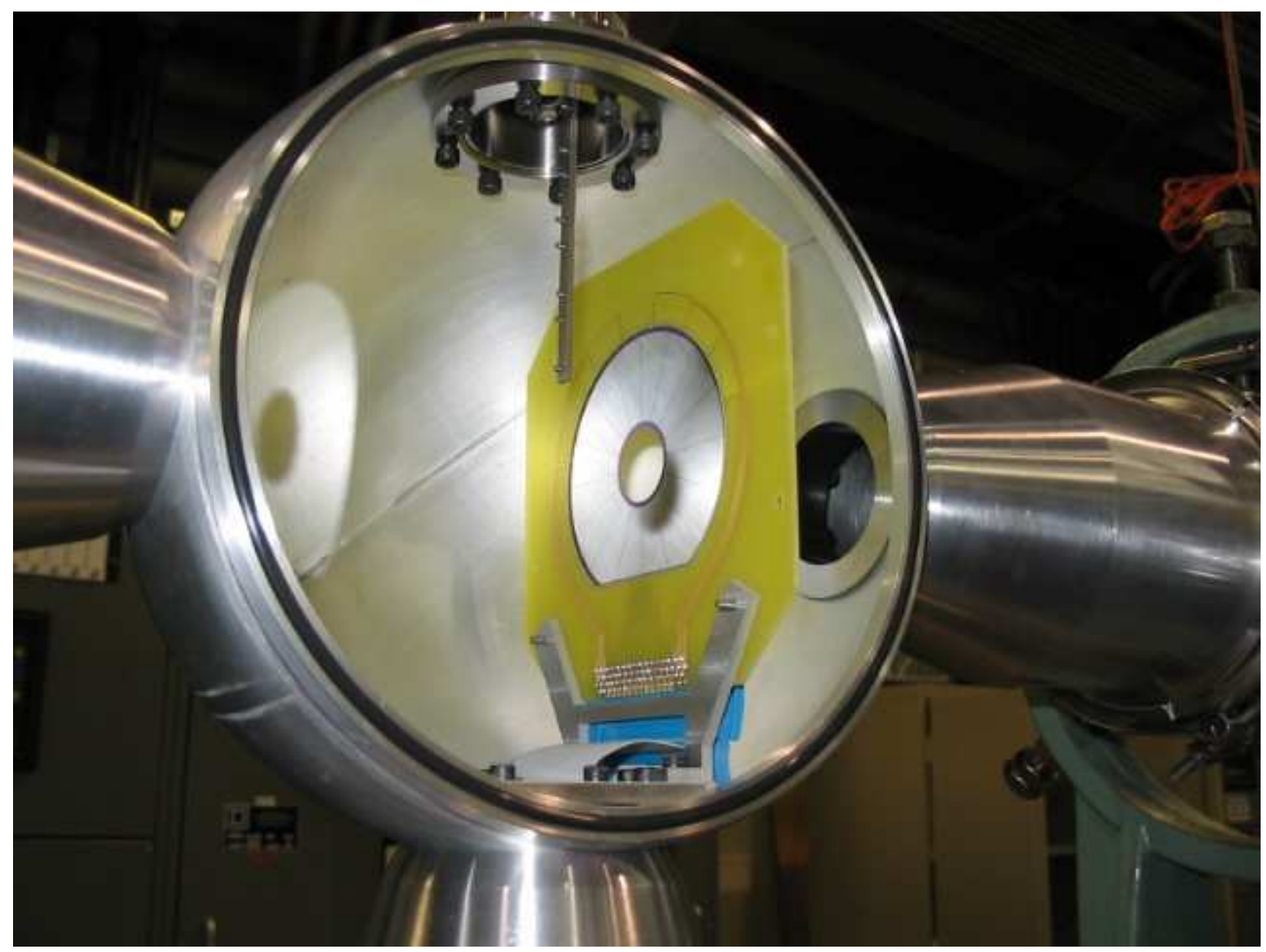

1 Figure 3. Photograph of one BAMBINO Si CD-S2 detector mounted in its target chamber. The

2 beam enters from the left, impinges on the Ti target (not shown) and passes through the hole in 3 the centre of the CD detector. 
ID 478

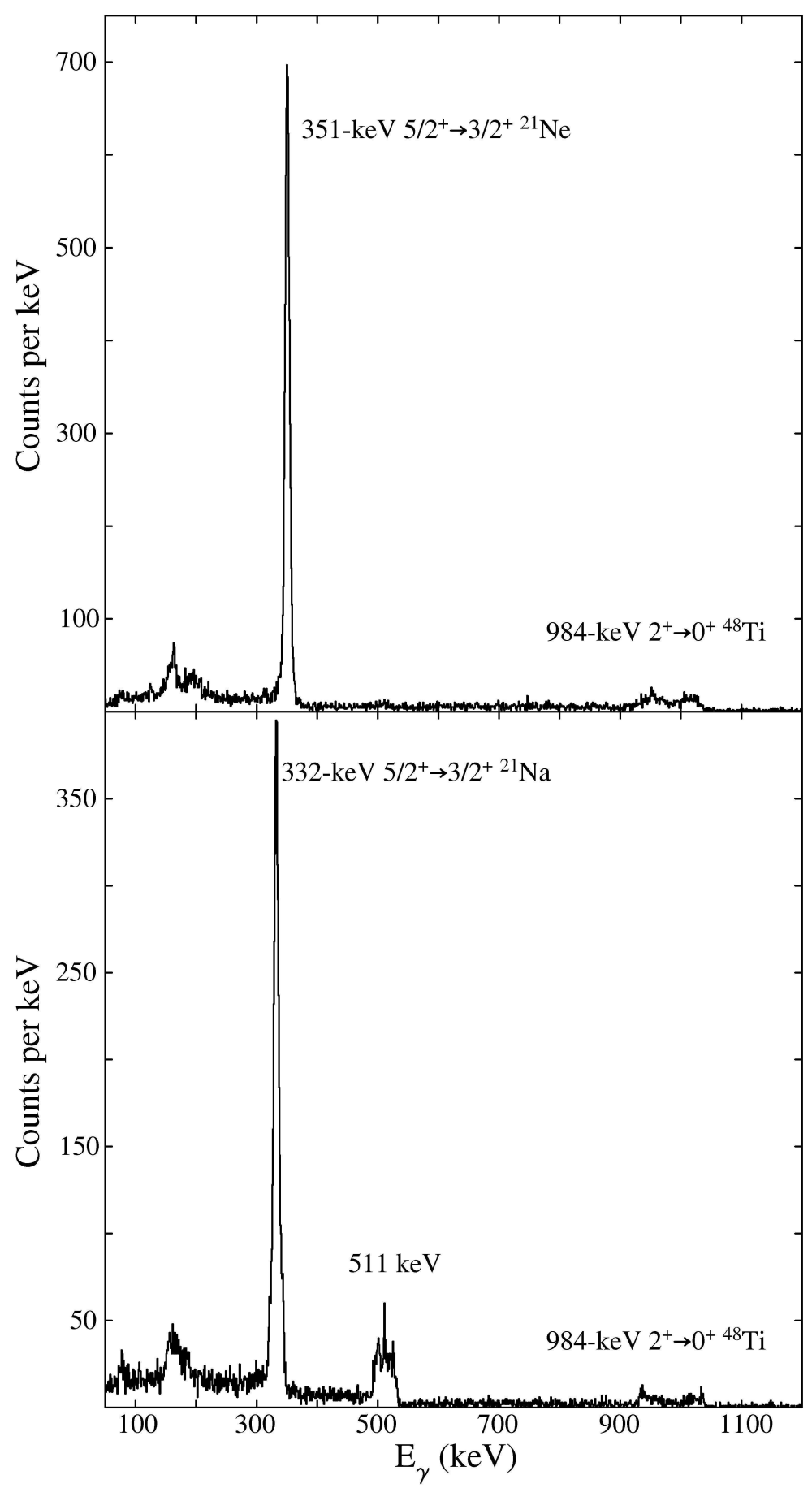

2 Figure 4. Portions of the $\gamma$-ray spectrum obtained from the Coulomb excitation of ${ }^{21} \mathrm{Ne}$ 3 (top) and ${ }^{21} \mathrm{Na}$ (bottom) on a target of ${ }^{\text {nat }} \mathrm{Ti}$ using BAMBINO and TIGRESS. A coincidence 4 condition with particles detected in BAMBINO has been applied, without background 5 subtraction and a preliminary Doppler correction for the scattered beam particles has been used. 